\title{
Perspectives on blood pressure by patients on haemo- and peritoneal dialysis
}

\section{Authors}

Nicole Evangelidis, MPhil ${ }^{1,2}$, Benedicte Sautenet, $\mathrm{PhD}^{3}$, Karine E Manera, MIPH ${ }^{1,2}$ Martin Howell, $\mathrm{PhD}^{1,2}$, Jonathan C Craig $\mathrm{PhD}^{4}$ Andrea K Viecelli, $\mathrm{PhD}^{5,6}$, Emma O’Lone, MBChB ${ }^{1,2}$, Nicole

Scholes-Robertson, BSc ${ }^{1,2}$, David W Johnson, $\mathrm{PhD}^{5,6,7}$, Yeoungjee Cho, $\mathrm{PhD}^{5,6,7}$, Charles Tomson, $\mathrm{DM}^{8}$, David C Wheeler, $\mathrm{PhD}^{9}$, Allison Tong, $\mathrm{PhD}^{1,2}$ for the SONG-HD and SONG-PD initiative

\section{Affiliations}

${ }^{1}$ Sydney School of Public Health, The University of Sydney, Sydney, New South Wales, Australia.

${ }^{2}$ Centre for Kidney Research, The Children's Hospital at Westmead, Sydney, New South Wales,

Australia.

${ }^{3}$ Service de Néphrologie, Hôpital Bretonneau, CHRU Tours, Université de Tours et Nantes,

SPHERE- INSERM 1246, Tours, France.

${ }^{4}$ College of Medicine and Public Health, Flinders University, Adelaide, Australia

${ }^{5}$ Department of Nephrology, Princess Alexandra Hospital, Brisbane, QLD, Australia.

${ }^{6}$ Australasian Kidney Trials Network, Centre for Health Services Research, University of

Queensland, Brisbane, QLD, Australia.

${ }^{7}$ Translational Research Institute, Brisbane, QLD, Australia.

${ }^{8}$ Renal Unit, Freeman Hospital, Newcastle, United Kingdom.

${ }^{9}$ Centre for Nephrology, University College London, London, UK.

This article has been accepted for publication and undergone full peer review but has not been through the copyediting, typesetting, pagination and proofreading process which may lead to differences between this version and the Version of Record. Please cite this article as doi: 10.1111/nep.13775 


\section{Corresponding author:}

Nicole Evangelidis

Centre for Kidney Research, The Children’s Hospital at Westmead, Westmead NSW 2145, Sydney, Australia

Tel: +61 466016200 | Email: nicole.evangelidis@sydney.edu.au

\section{Disclosure of interest}

The authors report no conflict of interest.

Short running title: Perspectives blood pressure dialysis 


\section{Abstract}

\section{Aim}

The management of blood pressure in patients requiring dialysis remains challenging and controversial. This study aimed to describe the perspectives of patients treated with peritoneal or haemodialysis regarding blood pressure, to inform patient-centred management.

\section{Methods}

We conducted a secondary thematic analysis of qualitative data from multiple datasets derived from the Standardised Outcomes in Nephrology (SONG) initiative. We extracted and analysed the responses of adult patients (aged 18 years or over) on haemodialysis and peritoneal dialysis, and their caregivers. Qualitative data were extracted from 26 focus groups, two international Delphi surveys and two consensus workshops completed as part of the SONG-Haemodialysis and SONGPeritoneal dialysis projects.

\section{Results}

Collectively, the studies involved 644 patients and caregivers from 86 countries. We identified four themes: helpless and incapacitated (including the subthemes of disabling and debilitating symptoms, limiting ability to work, fear of "crashes" - a sudden drop in blood pressure - forced to depend on others); dismissed and ignored (disregarded as a problem, lacking information, education and reassurance); escalating medication burden; and taking control for improved self-management (determining thresholds in fluid management, establishing a routine for proactive monitoring) 


\section{Conclusion}

Blood pressure symptoms are debilitating for patients on dialysis and exacerbated by a perceived lack of information about how to understand and manage these symptoms. More patient-centred management of blood pressure, particularly symptom-causing blood pressure, in patients on dialysis is likely to substantially improve patient satisfaction and outcomes.

Keywords: Blood pressure, haemodialysis, hypertension, hypotension, peritoneal dialysis 


\section{Introduction}

Blood pressure management in patients on dialysis remains a critical but controversial area. Intradialytic hypotension is a frequent complication that occurs in $10-20 \%$ of haemodialysis sessions and is associated with increased mortality, hospitalisation, myocardial dysfunction and distressing symptoms. ${ }^{1,2}$ Hypertension is diagnosed in up to $70-90 \%$ of patients on haemodialysis and peritoneal dialysis, associated with cardiovascular morbidity and mortality, and often poorly controlled. ${ }^{3,4}$ The major contributors to the pathogenesis of hypertension in people on dialysis are volume expansion and sodium excess. ${ }^{4}$

Recommendations for blood pressure management in dialysis are often extrapolated from guidelines for the general population and include multiple strategies, such as anti-hypertensive medications, fluid management and dietary sodium restriction. ${ }^{2,4}$ Specific blood pressure targets in dialysis are uncertain due to a lack of clinical trials. Recently a pilot study was conducted to investigate the optimal blood pressure target in dialysis and this may lay the groundwork for a fullscale trial. ${ }^{5}$ Observational data suggest that low blood pressure targets in dialysis may lead to worse outcomes, potentially due to higher risk of intradialytic hypotension. ${ }^{6,7}$ International guidelines state that there is insufficient evidence to recommend an explicit blood pressure target in dialysis.,9 Some experts have argued that blood pressure targets cannot be generalised to the dialysis population and must be individually assessed for each patient. ${ }^{2}$

These problems highlight the need for further evidence pertaining to the management of blood pressure in dialysis, which encompasses data on patient perspectives and preferences to support 
shared decision making and patient enablement to promote the uptake of recommended strategies. ${ }^{10-}$

12 This study aimed to describe the experiences and perspectives of patients on haemodialysis and peritoneal dialysis regarding blood pressure, to inform patient-centred management.

\section{Methods}

Context and sources of data

We conducted secondary analysis of qualitative data from multiple datasets from the Standardised Outcomes in Nephrology (SONG) initiative. The SONG initiative was established to develop core outcome sets to be reported in trials across the spectrum of kidney disease. The SONG-HD core outcomes (i.e. to be reported in all trials) are mortality, cardiovascular disease, vascular access, and fatigue $^{11}$. The SONG-PD core outcomes are mortality, technique survival, cardiovascular disease, infection and life participation. ${ }^{11}$ Blood pressure is in the second tier of outcomes i.e. outcomes that are "critically important to some stakeholder groups, to be reported in some trials"11 for both SONG-HD and SONG-PD.

Secondary analysis of qualitative data is the use of existing data that were collected to address a related or different research question, and is undertaken to maximise the use of the data collected that have not been analysed or used in the primary studies. ${ }^{13}$ We extracted and analysed the responses of adult patients (aged 18 years or over) on haemodialysis (HD) and peritoneal dialysis (PD) and caregivers that were specifically related to blood pressure. We used data from patients on both HD and PD in order to understand the different experiences and perspectives from both 
dialysis modalities. The data were extracted from 26 focus groups ${ }^{12,14}$, free text comments from two international Delphi surveys ${ }^{10,15}$ and two consensus workshops ${ }^{16}$ completed as part of the SONGHD and SONG-PD projects. This is all of the available data on dialysis from the SONG studies. These studies had a primary aim of describing patients' and health professionals' perspectives on critically important outcomes to be reported in trials in haemodialysis and peritoneal dialysis. Collectively, the studies involved 644 patients and caregivers from 86 countries. The institution review boards that provided ethics approval are listed in Supplementary File 1.

\section{$\underline{\text { Data extraction and analysis }}$}

We analysed the findings using thematic analysis and individually identified HD and PD data. All participant quotations were extracted from the original transcripts of the SONG-HD and SONG-PD focus groups (with nominal group technique) and consensus workshops, and from the online freetext responses in the SONG-HD and SONG-PD Delphi surveys. These were imported into HyperRESEARCH software (version 4.0.3; ResearchWare Inc., Randolph, Massachusetts, USA). Author N.E. reviewed the transcripts line by line and inductively identified and coded all concepts related to symptoms, decision-making, management, communication and education regarding blood pressure. We also compared themes by dialysis modality (haemodialysis and peritoneal dialysis), and with reference to hypertension and hypotension. The themes were discussed among N.E., B.S. and A.T. to ensure that they reflected the full range and depth of the data related to blood pressure.

\section{Results}


The characteristics of participants are summarised in Table 1. For SONG-HD, 58 patients and 24 caregivers from 2 countries participated in the nominal group technique study, 168 patients and 34 caregivers from 73 countries participated in the Delphi study, and seven patients and one caregiver from 2 countries were involved in the consensus workshop. For SONG-PD, 81 patients and 45 caregivers from three countries participated in the nominal group technique study, 177 patients and 36 caregivers from 68 countries participated in the Delphi study and 13 patients and 6 caregivers from 2 countries were involved in the consensus workshop.

We identified four themes: helpless and incapacitated, dismissed and ignored, escalating medication burden, and taking control for self-management. These themes and subthemes are described below with reference to HD or PD, as well as hypertension and hypotension where it was clearly specified. The themes involved in each analysis are shown in Figure 1. Selected quotations for each theme are provided in Table 2. Participant quotations that have been published in prior studies are referenced accordingly.

\section{Helpless and incapacitated}

Disabling and debilitating symptoms: For patients on HD and PD, the symptoms related to blood pressure were “incapacitating” and “completely debilitating”. Hypotensive episodes produced symptoms including feeling “dizzy”, “clammy”, "light-headed”, “sick,” and fainting all of which constrained their ability to function. Some patients reported fainting on public transport or requiring a wheelchair when exiting a dialysis centre as a result of a drop in blood pressure. A caregiver described blood pressure as determining the type of day he and his wife, who was on PD would 
have: “I’ve got number one as blood pressure, because that's what governs her. Her blood pressure's down, that's it, you're gone for the day, doesn't matter what the rest of them are. If her blood pressure's okay, then you have an okay sort of day” (PD). ${ }^{14}$ These symptoms and consequences, primarily related to hypotension in $\mathrm{HD}$ and $\mathrm{PD}$, made patients feel vulnerable and unable to function.

Limiting ability to work: The symptoms of low blood pressure impaired the ability to work in patients on HD and PD. In some instances, patients decided not to return to work due to hypotensive symptoms - "I would love to work part time as I miss the contact with the patients in the dental office I worked in. However, due to some tiredness and a low blood pressure problem it just would not work” (HD). Another patient almost fainted at work and had to lie down for part of her shift. She felt exasperated at the unpredictability of symptoms of low blood pressure that prevented her from working: “The frustrating thing is I can’t do anything about it, and I have no way of planning when it's going to happen....I can’t work lying flat on my back with my feet up the wall” (PD).

Fear of "crashes" (i.e. a sudden drop in blood pressure): Patients on HD perceived the symptoms of a drop in blood pressure as “frightening”, “scary”, “dangerous” and “risky.” They witnessed nurses looking "alarmed” when crashes occurred and observed other patients passing out in the dialysis unit. One patient explained that the fear of crashes prevented her from considering home dialysis: “I can’t do home dialysis, I don’t know how you guys can. I’m just too scared to do it. In case something goes wrong, and my blood pressure is a factor” (HD). One patient felt he could have 
“used more help understanding [hypotensive episodes]” (HD) so he could prevent several “dangerous” crashes occurring when he was learning how to use dialysis at home.

Forced to depend on others: Patients on HD felt they were entirely reliant on caregivers or health professionals to assist them with hypotensive episodes. These episodes often required immediate action, such as calling an ambulance, and could be stressful for everyone involved: "My husband's frantic, doesn’t want to leave me” (HD). In the dialysis centre, patients depended on nurses to raise their feet, push their head down or run a saline drip to manage hypotensive symptoms. Patients felt powerless in managing these symptoms: "There's nothing you could do. I couldn’t even speak" (HD).

\section{Dismissed and ignored}

Disregarded as a problem: Some patients on HD believed the risks and problems related to their blood pressure were ignored or mis-managed by health professionals. Some patients sought a different nephrologist in the hope of receiving better management of blood pressure and considered that their condition improved when closer attention was paid to their blood pressure symptoms: "When discussing with doctors they ignore my blood pressure levels and tell me there is no problem. After changing doctor it was fixed” (HD). Some felt they were automatically prescribed medication for high blood pressure, but they wanted to understand the cause: "Because most of the doctors, they hear my thing [hypertension], they just started to prescribe medicine. And this one and the last doctor, this specialist, I feel more confident, because he wants to find out a cause. He's the only person who did that.” (HD) 
Lacking information, education and reassurance: Patients on HD and PD felt hopeless and frustrated in being unable to obtain information about the causes, risks and treatment options related to their blood pressure symptoms. They had unresolved concerns and wanted to understand the cause of the fluctuating symptoms of blood pressure: "So for me, I've always wanted to know what it is that triggers the blood pressure behaviour and no one has ever, ever been able to explain it to me” (HD). Patients were discouraged when communicating with nephrologists, GPs and nurses who they perceived were unsure or unclear about blood pressure. For some patients, their blood pressure results were a “mystery” (HD) or being “investigated” (PD) without any resolution “Sometimes our [blood pressure] results are so varied that even doctors and nurses, and nurse practitioners themselves can’t understand - And it’s a big concern because it's really - it's a big part of your body” (HD). Some patients also felt powerless because they were dependent on doctors to translate their blood pressure results and were unsure how these results related to their physical symptoms and whether they were at increased risk of conditions, such as "heart disease" (HD) or “brain damage” (PD).

Patients felt confused about what type of medication was needed to manage their blood pressure and were concerned about nephrotoxic side effects which they believed were not communicated to them: "The medication that the doctors were giving me on there was just helping destroy my kidneys a lot faster than usual and I had no idea of this. But nobody ever told me that, how was I supposed to know?” Patients felt they were being “experimented on” in relation to blood pressure medication and would like to understand more about why their blood pressure can fluctuate. 


\section{Escalating medication burden}

The increasing number of blood pressure medications, in addition to other types of medication (such as for cholesterol and diabetes), elevated the burden of polypharmacy and patients felt overwhelmed: "the doctors keep piling medications" (HD). Patients felt “worried" that the medication burden would continue to increase and be an "ongoing problem" and were concerned about how it limited their lifestyle. Patients on PD explained they were taking three tablets each day for blood pressure or 7 or 8 different tablets in total: "The pill burden is actually increasing for me" (PD). Many patients experienced multiple conditions such as diabetes, high cholesterol and hypertension, which further added to their medications and intensified feelings of poor health.

\section{Taking control for self-management}

Determining thresholds in fluid management: Patients on HD and PD believed that understanding and controlling their fluid levels were "critical” and allowed for better control of blood pressure symptoms and could prevent hypotensive episodes: "So by taking control, this became a manageable thing” (HD). This led to a greater sense of empowerment and awareness of how their body functioned: “So you have to know yourself. You have to know your patterns” (HD). Some patients also believed that controlling fluids and limiting sodium in their diet would mean they would not require blood pressure medication. Being aware of fluid levels enabled better selfmanagement that could lead to a reduced need for medications and prevent the distressing symptoms of high or low blood pressure. 
Establishing a routine for proactive monitoring: Patients mostly on PD regularly monitored blood pressure and fluid levels, which they believed led to better self-management of blood pressurerelated symptoms, such as feeling “dizzy”, “light-headed” or “sick”. For many, recording results was a rewarding task and patients likened themselves to, "a school kid with your little book” (PD). It also felt satisfying and motivating to monitor results and view progress: "I test my blood pressure, I do it once a day, and I get really excited because lately it's been almost perfect” (PD). Many described the routine of monitoring and testing as a duty which was the "most important" and "vital” aspect of keeping their blood pressure under control: "good management is so important for a good outcome” (PD). For some, the task of monitoring blood pressure was shared and supported by caregivers: "My blood pressure is monitored several times a week by my wife” (PD). This also included setting phone reminders for taking blood pressure medication which enabled better adherence to medication. Many believed omitting to regularly observe blood pressure could have serious consequences for their health, such as increasing the risk of cardiovascular disease, as well as place other people at risk: “we do running sheets and everything ‘cause I’m thinking if I'm going through day by day with high blood pressure and I'm driving and I've got kids in the car and all sorts of things, well then, I'm putting everyone at risk” (HD).

\section{Discussion}

Patients on HD and PD experienced debilitating symptoms mainly related to hypotension, which caused them to depend on others, instigated fear and helplessness, and limited their ability to work, contributing to disempowerment (see Figure 1). Some patients felt that clinicians did not acknowledge or address fluctuating blood pressure nor explain the cause of their blood pressure 
symptoms. They were concerned about taking multiple medications because this increased their sense of powerlessness. For patients on HD and PD, they believed fluid management was crucial for self-management of symptoms. For patients on PD, establishing a routine for monitoring their blood pressure by using log books or blood pressure monitoring machines at home was an important way to gain control, manage symptoms and increase empowerment (Figure 1). We did not identify any themes that were specific based upon HD location (i.e. home HD vs. in-centre HD).

Our findings reflect the results of other studies about dialysis-related symptoms, which have also highlighted the distressing impact of hypotensive episodes. ${ }^{17-19}$ In a study of 550 patients on haemodialysis, intradialytic hypotension was the second most common symptom, being reported by $76 \%$ of patients. ${ }^{17}$ Studies of hypertension in the general population have also reported dissatisfaction with polypharmacy and a lack of information from health professionals being associated with non-adherence to treatment. ${ }^{20}$ The benefits of self-management practices, such as home blood pressure monitoring, have previously been shown and echo our findings that patients feel empowered by self-management and monitoring their own blood pressure and fluid levels. ${ }^{18,21-}$ 23

This study is based on a comprehensive data set, including perspectives of patients and caregivers from more than 86 countries. It has generated additional insights specifically into the challenges of managing blood pressure and its impact on patients that were not analysed or reported in the primary studies. There are some potential limitations; the source studies were conducted in mostly English-speaking participants from high-income countries. ${ }^{10-12,14,15}$ We also acknowledge the potential limitations in secondary analysis of qualitative data. The depth and breadth of the data 
may be limited as specific probing questions related to blood pressure may not have been included in the original studies. We restricted this study to patients on dialysis, however, we acknowledge it is important to investigate the perspectives of patients with CKD who are not on dialysis.

Our findings highlight opportunities to improve the management and awareness of blood pressure in dialysis through increased education and shared decision making. Educational interventions to support self-management in patients on HD have demonstrated improvements in dialysis-related symptoms (such as interdialytic weight gain), adherence to treatment, increased self-efficacy and self-management skills, and have also been associated with lower levels of anxiety and depression. ${ }^{24,25}$ Shared decision aids have been used successfully in interventions supporting patient preferences about choice of dialysis modality, ${ }^{26}$ but their use in the context of blood pressure is not well-understood due to a lack of research in this area.

Further research on blood pressure should take into account patient viewpoints and what they may be willing to "trade off" in order to improve quality of life. Discrete choice experiments may provide further insight into understanding and weighing up the long-term benefits associated with blood pressure management against adverse outcomes. Similar patient preference studies have already been performed in nephrology reporting preferences for dialysis modality, ${ }^{27}$ treatment in kidney transplantation, ${ }^{28}$ end of life care, ${ }^{29}$ management of anaemia in $\mathrm{HD},{ }^{30}$ and relocation for patients on dialysis in remote communities. ${ }^{31}$

Blood pressure symptoms are overwhelming for patients on dialysis and are exacerbated by a perceived lack of information about the cause and management of these symptoms, which are often 
unpredictable and frightening. Improved decision-making between patients, caregivers and health professionals may improve satisfaction with dialysis treatment and allow for patient-centred management of symptoms. 


\section{References}

1. Flythe JE, Xue H, Lynch KE, Curhan GC, Brunelli SM. Association of mortality risk with various definitions of intradialytic hypotension. J Am Soc Nephrol. 2015;26(3):724-734.

2. Agarwal R, Flynn J, Pogue V, Rahman M, Reisin E, Weir MR. Assessment and management of hypertension in patients on dialysis. J Am Soc Nephrol. 2014;25(8):16301646.

3. Levin A. Clinical epidemiology of cardiovascular disease in chronic kidney disease prior to dialysis. Semin Dial. 2003;16(2):101-105.

4. Sarafidis PA, Persu A, Agarwal R, et al. Hypertension in dialysis patients: a consensus document by the European Renal and Cardiovascular Medicine (EURECA-m) working group of the European Renal Association - European Dialysis and Transplant Association (ERA-EDTA) and the Hypertension and the Kidney working group of the European Society of Hypertension (ESH). J Hypertens. 2017;35(4):657-676.

5. Miskulin DC, Gassman J, Schrader R, et al. BP in Dialysis: Results of a Pilot Study. J Am Soc Nephrol. 2018;29(1):307-316.

6. Davenport A, Cox C, Thuraisingham R. Achieving blood pressure targets during dialysis improves control but increases intradialytic hypotension. Kidney Int. 2008;73(6):759-764.

7. Tentori F, Hunt WC, Rohrscheib M, et al. Which targets in clinical practice guidelines are associated with improved survival in a large dialysis organization? J Am Soc Nephrol. 2007;18(8):2377-2384.

8. Daugirdas JT, Depner TA, Inrig J, et al. KDOQI Clinical Practice Guideline for Hemodialysis Adequacy: 2015 Update. Am J Kidney Dis. 2015;66(5):884-930. 
9. Levin NW, Kotanko P, Eckardt KU, et al. Blood pressure in chronic kidney disease stage 5D-report from a Kidney Disease: Improving Global Outcomes controversies conference. Kidney Int. 2010;77(4):273-284.

10. Evangelidis N, Tong A, Manns B, et al. Developing a Set of Core Outcomes for Trials in Hemodialysis: An International Delphi Survey. Am J Kidney Dis. 2017;70(4):464-475.

11. Tong A, Manns B, Wang AYM, et al. Implementing core outcomes in kidney disease: report of the Standardized Outcomes in Nephrology (SONG) implementation workshop. Kidney Int. 2018;94(6):1053-1068.

12. Urquhart-Secord R, Craig JC, Hemmelgarn B, et al. Patient and Caregiver Priorities for Outcomes in Hemodialysis: An International Nominal Group Technique Study. Am J Kidney Dis. 2016;68(3):444-454.

13. Tate JA, Happ MB. Qualitative Secondary Analysis: A Case Exemplar. J Pediatr Health Care. 2018;32(3):308-312.

14. Manera KE, Johnson DW, Craig JC, et al. Patient and Caregiver Priorities for Outcomes in Peritoneal Dialysis: Multinational Nominal Group Technique Study. Clin J Am Soc Nephrol. 2019;14(1):74-83.

15. Manera KE, Tong A, Craig JC, et al. An international Delphi survey helped develop consensus-based core outcome domains for trials in peritoneal dialysis. Kidney Int. 2019.

16. Tong A, Manns B, Hemmelgarn B, et al. Establishing Core Outcome Domains in Hemodialysis: Report of the Standardized Outcomes in Nephrology-Hemodialysis (SONGHD) Consensus Workshop. Am J Kidney Dis. 2017;69(1):97-107.

17. Caplin B, Kumar S, Davenport A. Patients' perspective of haemodialysis-associated symptoms. Nephrol Dial Transplant. 2011;26(8):2656-2663. 
18. Juergensen E, Wuerth D, Finkelstein SH, Juergensen PH, Bekui A, Finkelstein FO. Hemodialysis and Peritoneal Dialysis: Patients’ Assessment of Their Satisfaction with Therapy and the Impact of the Therapy on Their Lives. Clin J Am Soc Nephrol. 2006;1(6):1191-1196.

19. Kuipers J, Oosterhuis JK, Paans W, et al. Association between quality of life and various aspects of intradialytic hypotension including patient-reported intradialytic symptom score. BMC Nephrol. 2019;20(1):164.

20. Marshall IJ, Wolfe CDA, McKevitt C. Lay perspectives on hypertension and drug adherence: systematic review of qualitative research. BMJ. 2012;345:e3953.

21. Francois K, Bargman JM. Evaluating the benefits of home-based peritoneal dialysis. Int $J$ Nephrol Renovasc Dis. 2014;7:447-455.

22. Agarwal R, Andersen MJ, Bishu K, Saha C. Home blood pressure monitoring improves the diagnosis of hypertension in hemodialysis patients. Kidney Int. 2006;69(5):900-906.

23. Jolles EP, Padwal RS, Clark AM, Braam B. A Qualitative Study of Patient Perspectives about Hypertension. ISRN Hypertension. 2013;2013:10.

24. Griva K, Nandakumar M, Ng J-aH, Lam KFY, McBain H, Newman SP. Hemodialysis Selfmanagement Intervention Randomized Trial (HED-SMART): A Practical Low-Intensity Intervention to Improve Adherence and Clinical Markers in Patients Receiving Hemodialysis. Am J Kidney Dis. 2018;71(3):371-381.

25. Mason J, Khunti K, Stone M, Farooqi A, Carr S. Educational interventions in kidney disease care: a systematic review of randomized trials. Am J Kidney Dis. 2008;51(6):933-951.

26. Finderup J, Jensen JKD, Lomborg K. Developing and pilot testing a shared decision-making intervention for dialysis choice. J Ren Care. 2018. 
27. Morton RL, Snelling P, Webster AC, et al. Dialysis modality preference of patients with CKD and family caregivers: a discrete-choice study. Am J Kidney Dis. 2012;60(1):102-111.

28. Howell M, Wong G, Rose J, Tong A, Craig JC, Howard K. Patient Preferences for Outcomes After Kidney Transplantation: A Best-Worst Scaling Survey. Transplantation. 2017;101(11):2765-2773.

29. Clark MD, Szczepura A, Gumber A, Howard K, Moro D, Morton RL. Measuring trade-offs in nephrology: a systematic review of discrete choice experiments and conjoint analysis studies. Nephrol Dial Transplant. 2018;33(2):348-355.

30. Hauber B, Caloyeras J, Posner J, Brommage D, Tzivelekis S, Pollock A. Hemodialysis patients’ preferences for the management of anemia. BMC Nephrol. 2017;18(1):253.

31. Tonelli M, Molzahn AE, Wiebe N, et al. Relocation of remote dwellers living with hemodialysis: a time trade-off survey. Nephrol Dial Transplant. 2015;30(10):1767-1773. 


\section{CONFLICT OF INTEREST STATEMENT}

The results presented in this paper have not been published previously. This is applicable to all authors. 


\section{TABLES AND FIGURES}

Table 1. Characteristics of participants by data source.

Table 2. Selected illustrative quotations for each theme.

Figure 1. Cross-tabulation of themes by dialysis modality and blood pressure symptoms. 
Tahle 1. Characteristics of participants by data source, n (\%).

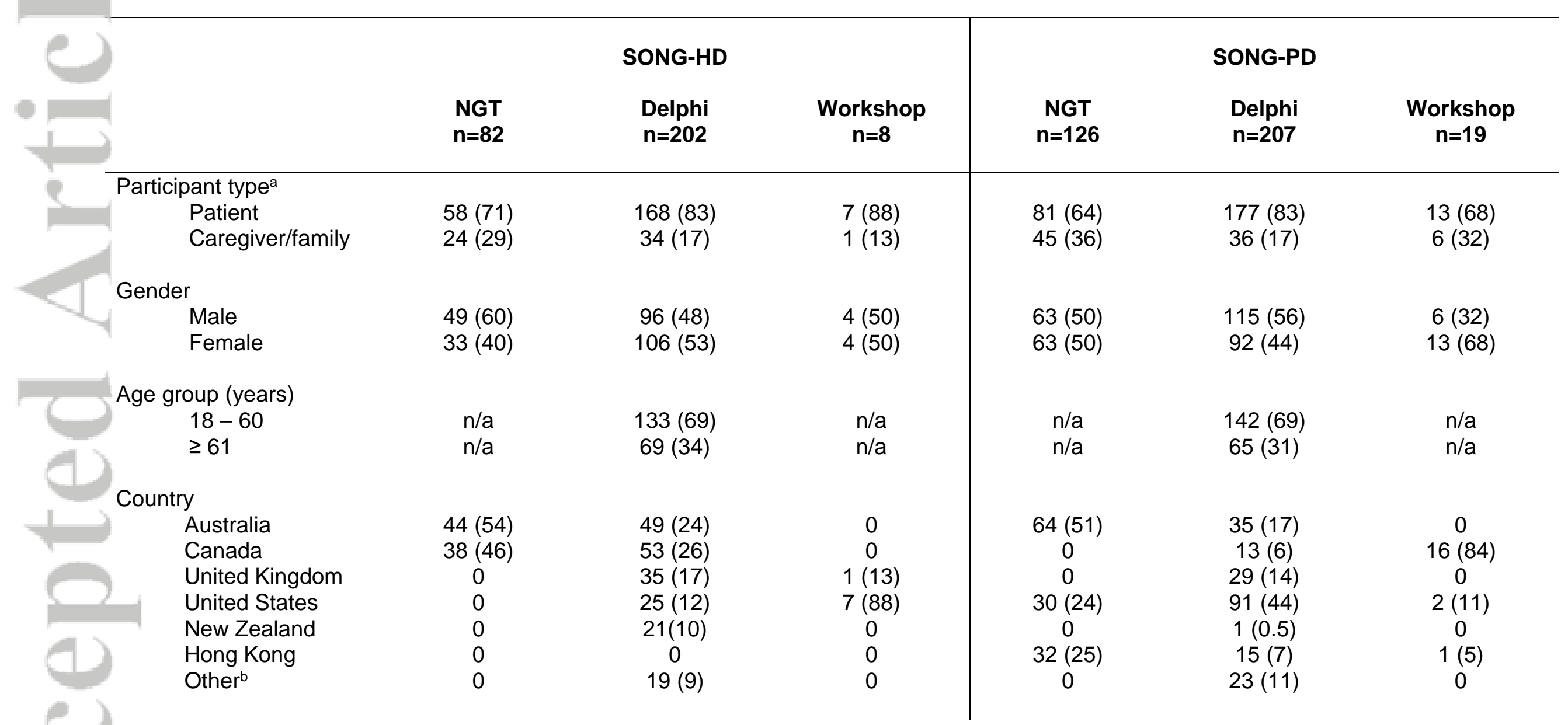


HD modality (patient only) ${ }^{c}$ In-centre

SOI G: Standardised Outcomes in Nephrology; HD: Haemodialysis; PD: Peritoneal dialysis; NGT: Nominal Group Technique; Delphi: a sequential, consensus-based survey method. me have multiple roles. '⿳⺈冂大 ${ }^{2}$ ther includes 15 countries: Brazil, China, Czech Republic, Denmark, Egypt, Germany, India, Indonesia, Ireland, Italy, the Netherlands, Nigeria, Philippines, Romania and Spain. ${ }^{c}$ Percentages do not add up to 100 due to undisclosed responses (excluded). 
Tahle 2. Selected illustrative quotations for each theme.

\section{Helpless and incapacitated}

\section{Dis zbling and debilitating symptoms}

- 'It's incapacitating. You physically feel like you'd just pass out and I've seen it happen to me both when it goes low, and when it went high - low is likely you just get dizzy. High is where you get the dizziness but you also get the nausea as well, so it's completely debilitating." (HD)

- "And I've been sent down to my ride in a wheelchair, 'cause my blood pressure's low." (HD)

- 'I've got number one as blood pressure, because that's what governs her. Her blood pressure's down, that's it, you're gone for the day, doesn't matter what the rest of them are. If her blood pressure's okay, then you have an okay sort of day" (PD)

- "We've been on trains and she's had a blackout." (PD)

\section{im iting ability to work}

- "I would love to work part time as I miss the contact with the patients in the dental office I worked in. However, due to some tiredness and a low blood pressure problem is just would not work" (HD)

- "The frustrating thing is I can't do anything about it, and I have no way of planning when it's going to happen [drop in blood pressure]. I've turned up for work at night shift, have been doing the restricted drug count that we have to do at the start and at the end of our shift, and gone to my co-worker oh, got to lay down otherwise I'm going to faint. Lie down, put my feet up against the wall and feel perfectly fine while I'm laying like that, but I can't work lying flat on my back with my feet up the wall." (PD). 
- This is THE MOST SCARY THING about dialysis." (HD)

- “I can't do home dialysis. I don't know how you guys can. I'm just too scared to do it. In case something goes wrong, and my blood pressure is a factor. I mean, the girls work out how much fluid they take off me, but very often, I still - like I got up the .. thinking one was fine. Stood up and it was like 55/40 $<$ laughs $>$ and - yeah. I wouldn't be able to handle that at home. Just let them do it." (HD)

- $\quad$ "Can be dangerous, happened a lot as I was learning. Could have used more help understanding it, I was doing it alone and had to stop myself from passing out." (HD)

- " $\quad$ Realize it is very important and have seen alarm in nurses when it has happened to other people." (HD)

\section{For sed to depend on others}

- "And before I could do anything, I had to call for my husband then I passed out and then ambulance came and I was - yeah - quite critical, so - yeah. That's the first time in four years. My husband's frantic, doesn't wanna leave me." (HD)

"Can be dangerous! [drop in blood pressure] Dialysis nurses are quick to respond, but my brother's a big guy and his chair was old and they couldn't get his head down as fast as they'd liked to." (HD)

- When I used to get on the machine every 15 minutes, my blood pressure just drops. [My wife] took it about two weeks ago and I was 80 over 60. And then you just feel yourself crashing. There's nothing you could do. I couldn't even speak and the girls just looked at me and go, "Oh alright. There's something wrong with you," so they just run the saline back into me for a while." (HD) 


\section{Disregarded as a problem}

- When discussing with doctors they ignore my blood pressure levels and tell me there is no problem. After changing doctor it was fixed. It indicates inconsistency of what is acceptable and not. Since my blood pressure is at a good level, I feel a lot better and relaxed." (HD)

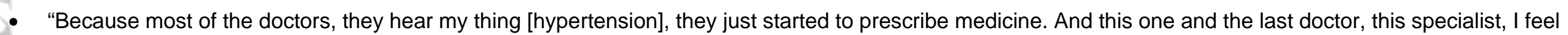
more confident, because he wants to find out a cause. He's the only person who did that." (HD)

"My blood pressure has been ignored for years despite my pleas for help and long term high blood pressure. Now I have an enlarged heart and empty pockets. I don't exactly feel well cared for. I feel left to die slowly while I become bankrupt." (HD)

\section{Lacking information, education and reassurance}

- "So for me, I've always wanted to know what it is that triggers the blood pressure behaviour and no one has ever, ever been able to explain it to me." (HD)

- ' 'Sometimes our [blood pressure] results are so varied that even doctors and nurse, and nurse practitioners themselves can't understand - And it's a big concern because it's really - it's a big part on your body." (HD).

- "The medication that the doctors were giving me on there was just helping destroy my kidneys a lot faster than usual and I had no idea of this. When I got here and started going with my kidney doctor now, he said, Well, if you've been taking this medication, your kidneys wouldn't be in such bad shape. But nobody ever told me that, how was I supposed to know?" (HD)

"I don't know why happening, that blood pressure, because it's a risk when you have high blood pressure- Risk of brain damage or - that's my opinion, you 
know, you go high high and then oh, what's happening? You don't know what's happening to your body." (PD)

\section{Escalating medication burden}

- "He's more worried, like there'll be another set of medications prescribed while the doctors to take in this medication you go such and such, this is a bit - the doctors keep piling medications." (HD)

- "An ongoing major problem as too much BP medication limits lifestyle." (PD)

- "There's also the number of medications, so the pill burden is actually increasing for me." (PD).

\section{Taking control for self-management}

\section{etermining thresholds in fluid management}

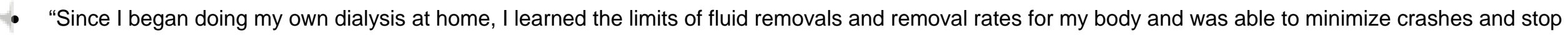
them much sooner as I pushed my dry weight. So by taking control, this became a manageable thing. Often protocols and doctors' orders for dry weights are aggressive and cause unnecessary crashing and cramping." (HD).

" "So you have to know yourself. You have to know your patterns." (HD)

\section{Establishing a routine for proactive monitoring}

- "Yeah, routine. You got your little, you feel like a school kid with your little book" (PD)

"Good management is so important for a good outcome" (PD)

"My blood pressure is monitored several times a week by my wife" (PD) 
"We do running sheets and everything 'cause I'm thinking if I'm going through day by day with high blood pressure and I'm driving and I've got kids in the car and all sorts of things, well then, l'm putting everyone at risk" (HD)

HD: Haemodialysis; PD: Peritoneal dialysis; "Crashes": Sudden drop in blood pressure. 
Figure 1. Cross-tabulation of themes by dialysis modality and blood pressure symptoms.

HD PD

Fear of crashes
Disabling and debilitating symptoms

HD: Haemodialysis; PD: Peritoneal dialysis; "Crashes": sudden drop in blood pressure. 